\title{
Risk of persistent and recurrent cervical neoplasia following incidentally detected adenocarcinoma-in-situ
}

Short title: Health outcomes after an incidental diagnosis of AIS

1. Dr Aime MUNRO PhD; Fremantle, Western Australia, Australia. Institute of Health Research, University of Notre Dame Australia.

2. Professor Jim CODDE PhD; Fremantle, Western Australia, Australia. Institute of Health Research, University of Notre Dame Australia.

3. Associate Professor Katrina SPILSBURY PhD; Bentley, Western Australia, Australia. Centre for Population Health Research, Curtin University.

4. Mrs Nerida STEEL; Perth, Western Australia, Australia. WA Cervical Cancer Prevention Program, WA Health.

5. Clinical Professor Colin J R. STEWART MBBS; Crawley, Western Australia, Australia. School of Women's and Infants' Health, University of Western Australia.

6. Dr Stuart G. SALFINGER MBBS; Subiaco, Western Australia, Australia. St. John of God Hospital Bendat Family Comprehensive Cancer Centre.

7. Dr Jason TAN MBBS; Subiaco, Western Australia, Australia. St. John of God Hospital Bendat Family Comprehensive Cancer Centre.

8. Dr Ganendra R. MOHAN MBBS; Subiaco, Western Australia, Australia. St. John of God Hospital Bendat Family Comprehensive Cancer Centre.

9. Professor Yee LEUNG MBBS; Crawley, Western Australia, Australia. School of Women's and Infants' Health, University of Western Australia.

10. Professor James SEMMENS PhD; Bentley, Western Australia, Australia. Centre for Population Health Research, Curtin University.

11. Professor Peter O'LEARY PhD, Bentley, Western Australia, Australia. School of Health Sciences, Curtin University

12. Associate Professor Vincent WILLIAMS PhD, Bentley, Western Australia, Australia. School of Biomedical Sciences, Curtin University.

13. Dr Paul A. COHEN MD; Subiaco, Western Australia, Australia. St. John of God Hospital Bendat Family Comprehensive Cancer Centre. 


\section{Acknowledgement:}

We gratefully acknowledge the Data Linkage Branch and Data Custodians of the Cervical Screening Registry of Western Australia, WA Cancer Registry and the Death Notification System (Western Australian Government Department of Health) for providing data for this project. Finally, a special thank you must be extended to our patients.

\section{Corresponding author's details:}

Dr Aime Munro

Institute of Health Research

University of Notre Dame Australia (Fremantle)

Fremantle WA Australia 6153

(M) +61404990169

(E) aime.munro@nd.edu.au

\section{Conflicts of Interest notification}

$\mathrm{N}$ Steel is employed by the WA Cervical Cancer Prevention Program that is responsible for maintaining and operating the Cervical Screening Register of WA 


\begin{abstract}
Background.

Adenocarcinoma-in-situ (AIS) of the uterine cervix is a precursor to cervical adenocarcinoma and may co-exist with both adenocarcinoma and high-grade squamous dysplasia (cervical intraepithelial neoplasia (CIN) 2 and 3). Up to $60 \%$ of AIS lesions are detected incidentally following excisional biopsies performed for the treatment of CIN 2/3. To date there are no data regarding risk factors for persisting or progressive cervical neoplasia in these patients.
\end{abstract}

Objective.

To investigate patient outcomes following incidentally detected cervical AIS after loop electrosurgical excision procedure (LEEP) or cold knife cone (CKC) biopsy performed for the treatment of high-grade cervical intraepithelial neoplasia (CIN).

Study design.

A retrospective, population-based cohort study of Western Australian patients with an incidental diagnosis of AIS between 2001 and 2012. Primary outcomes were persistent or recurrent CIN 2/3 and or AIS, and invasive adenocarcinoma during follow-up (<12 months) and surveillance ( $\geq 12$ months) periods.

Results.

The cohort comprised 298 patients, with 228 (76.5\%) treated initially by LEEP and 70 (23.5\%) treated by CKC biopsy. The mean age was 31.2 years (range 18 to 68 years) and the median length of follow-up was 2.4 years (range 0.3 to 12.2 years). Overall, $11(3.7 \%)$ patients had CIN 2/3, $23(7.7 \%)$ had AIS and $3(1.0 \%)$ had adenocarcinoma diagnosed during the follow-up and surveillance periods. Age over 30 years, pure AIS lesions and larger lesions (>8mm) were associated with a greater risk of disease persistence or recurrence.

Conclusion(s).

Following the incidental detection of AIS, age $>30$ years, pure AIS lesions and lesions $>8 \mathrm{~mm}$, were significantly associated with disease persistence/recurrence. In younger women, incidentally detected AIS which co-exists with $\mathrm{CIN} \mathrm{2/3}$ and is $<8 \mathrm{~mm}$ extent with clear margins may not require re-excision. 


\section{INTRODUCTION}

Adenocarcinoma-in-situ (AIS) of the uterine cervix is a precursor to cervical adenocarcinoma ${ }^{1,2}$ and may co-exist with both adenocarcinoma and high-grade squamous dysplasia (cervical intraepithelial neoplasia $(\mathrm{CIN}) 2$ and 3$)^{3-8}$. Following the diagnosis of AIS on screening cytology or cervical biopsy, an excisional procedure such as a cold knife cone (CKC) biopsy or loop electrosurgical excision procedure (LEEP) is mandatory to exclude invasive disease ${ }^{3,6,7,9}$. Because AIS is most prevalent in women under forty years of age such procedures may be considered adequate treatment in women desiring fertility preservation providing margins are clear ${ }^{5,8-13}$.

Many patients with AIS are asymptomatic with the diagnosis often being made on routine cervical screening ${ }^{11,14-17}$. However, up to $60 \%$ of AIS lesions are detected incidentally following clinical examination for CIN 2/3 ${ }^{15-18}$. The 2012 Canadian Colposcopic Management of Abnormal Cervical Cytology and Histology clinical practice guidelines recommend careful assessment of margins when AIS is found incidentally in a LEEP biopsy performed for $\mathrm{CIN}^{3}$. These guidelines do not advocate a repeat excisional procedure providing that the glandular (AIS) lesion is small, margins are clear, and fertility preservation is desired by the patient ${ }^{3}$. However, lesion size is not specified and therefore it is unclear what is meant by 'small'. More generally, to our knowledge there are no data determining risk factors for persisting or progressive cervical neoplasia in these patients.

Since data determining the optimal management of women with incidentally detected AIS are lacking ${ }^{18}$, in the current study we aimed to investigate the risk of persistent/recurrent cervical neoplasia in such women in a large population-based 
cohort in Western Australia (WA).

\section{MATERIALS AND METHODS}

\section{Data sources and linkage procedure}

The Western Australian Data Linkage System (WADLS) is an internationally recognized and validated data linkage system that facilitates person-level linkage of records in various correlates state health-related population-based data sets ${ }^{19,20}$. The Cervical Screening Registry (CSR) of WA is legislatively required to record all cervical test results (HPV, cytology and histology) for WA residents. The CSR is an 'opt off' Register with less than $0.05 \%$ of women requesting the removal of their demographic information and cervical test results ${ }^{5,21}$. The CSR of WA has been operational since 1991 and utilizes many quality assurance processes to ensure data completeness. The Cancer Registry of WA has been operational since 1982 and records all cases of cervical cancer.

The WADLS provided a de-identified extraction of linked data from the CSR of WA (2001-2012), Cancer Registry of WA (2001-2012) and WA Death Registry (20012012) for all women with biopsy confirmed AIS. Each record in the linked datasets contained the encrypted patient identification and age. Data fields in the CSR of WA included cervical screening data, type of procedure (e.g. cervical punch biopsy, LEEP, CKC biopsy or hysterectomy), patient age at time of excisional treatment, margin status, and depth of the surgical specimen (measured macroscopic extent along the cervical canal). Data fields in the Death records included: date of death.

Study data were obtained following approval from the Curtin University Human 
Research Ethics Committee (HREC) (project number: HR 86/2012), the Western Australian Department of Health HREC (project number: 2012/49) and the University of Notre Dame Australia HREC (project number: 016031F).

\subsection{Selection of patients}

Patients aged 18 years or older who had histologically confirmed AIS recorded in the CSR of WA from 2001 to 2012 were identified. Patients were then excluded if:

- AIS was identified on cervical screening or on punch biopsy prior to the excisional treatment.

- they had a history of previous treatment for high-grade CIN or cervical carcinoma.

- the AIS was initially treated with hysterectomy.

Cervical cytology results were classified according to the Australian Modified Bethesda System $2004^{9}$. Patient age at the time of excisional treatment was classified as $\leq 30$ years or $>30$ years. Postcode of residence was used to assign a socioeconomic level using the Australian Bureau of Statistics 2006 Socio Economic Indexes for Areas (SEIFA). Patients underwent excisional treatment procedures, CKC biopsy, LEEP or hysterectomy at the recommendation and advice provided by the treating specialist.

\section{Histopathology findings}

All histopathology reports were reviewed to confirm the type of excisional biopsy (CKC biopsy or LEEP), the number of surgical specimens, depth of the specimen, presence of concurrent high-grade $\mathrm{CIN}$ and resection margin status. The number of surgical specimens was classified as 1 or $>1$, the latter including both intentional two-stage procedures (LEEP followed by 'top hat' endocervical sampling) as well as technically 
difficult procedures which resulted in multiple, fragmented or incomplete specimens. Margin status was considered 'positive' if any margin (ectocervical, endocervical or deep/circumferential) was involved by CIN 2/3 and/or AIS, 'negative' if all margins were histologically clear of in situ disease, and 'indeterminate' if margins could not be assessed or were not documented. The extent of AIS was determined whenever possible from the initial histopathology reports in one of two ways. In some specimens, the maximal extent of AIS was specifically documented while in other cases the number of tissue blocks demonstrating AIS was recorded; in the latter situation, AIS extent was estimated assuming a 'standard' block width of $2.5 \mathrm{~mm}$ multiplied by the total number of involved blocks ${ }^{22}$. In the event that the lesion size and/or number of positive blocks was not reported, the specimens were recorded as lesion size not reported'. For those cases with reported AIS extent, the lesion size was categorized as i) $<1.5 \mathrm{~mm}$, ii) $\geq 1.5$ to $\leq 2.0 \mathrm{~mm}$, iii) $>2.0$ to $\leq 2.5 \mathrm{~mm}$ iv) $>2.5$ to $\leq 5 \mathrm{~mm}, \mathrm{v}$ ) $>5$ to $\leq 8 \mathrm{~mm}$, or vi) $>8 \mathrm{~mm}$.

\section{Follow-up}

Subsequent follow-up of patients potentially included cytological review, repeat CKC or LEEP biopsy and/or hysterectomy. The follow-up period was defined as the date of the initial excisional procedure to the date of the last follow-up procedure (e.g. cervical cytology, biopsy or hysterectomy) or death.

\section{Principal outcomes}

The principal outcomes to be ascertained were i) persistence of CIN 2/3 or AIS, or a diagnosis of adenocarcinoma during the follow-up period (defined as $<12$ months after initial diagnosis), and ii) recurrence of CIN $2 / 3$ or AIS, or a diagnosis of 
adenocarcinoma within the surveillance period (defined as $\geq 12$ months after initial diagnosis).

\section{Statistics}

Kaplan-Meier graphs were constructed to investigate the survivorship function (time from initial excisional procedure until biopsy-confirmed high-grade cervical neoplasia persistence or recurrence), and log-rank tests were used to assess equality of the survivorship function. Proportional hazards models were constructed after simultaneously adjusting for multiple factors to investigate the relative rate (hazard ratio) of having, or subsequently, developing a high-grade cervical lesion post initial treatment. Models were constructed using purposeful selection of covariates age at treatment, type of excisional procedure (CKC biopsy or LEEP), margin status, lesion size, and depth of excised tissue.

Statistical significance was determined as a p-value $<0.05$ and the $95 \%$ confidence intervals $(\mathrm{Cl})$ for hazard rate ratios were calculated. Violation of the proportionalhazard assumptions was assessed and biologically plausible interaction terms between variables were tested. STATA/IC 13.0 (STATA Corporation, College Station, TX, USA) was used for statistical analysis.

\section{RESULTS}

Study cohort

During the study period 345 patients undergoing excisional treatment for CIN 2/3 had an incidental finding of AIS. Forty-seven patients were excluded from further analysis because screening history or follow-up was not available, or because the patients 
underwent hysterectomy (Figure 1). For the remaining 298 patients, the mean age was 31.2 years (range 18 to 68 years) and median follow-up interval was 2.4 years (range 0.3 to 12.2 years). Baseline patient demographic showed subjects categorized according to measures of Social advantage or disadvantage were evenly distributed. (Table 1).

AIS lesion size could be ascertained in 152 (51\%) cases (Table 1) of which 74 were directly measured and 78 determined from the number of involved blocks. The median lesion size was $2.5 \mathrm{~mm}$ (range 0.4 to $22.5 \mathrm{~mm}$ ) (Table 1). Second excisional procedure specimens revealed a range of histopathology outcomes among subjects with persistent disease when compared to initial margin status (Table 2).

Follow-up period (< 12 months post treatment)

In the 12 months following the initial CKC or LEEP biopsy, $108(36.2 \%)$ patients had a second excisional procedure; 66 were due to positive margins in the initial excision specimen and 42 were due to high grade cervical cytology and/or cervical biopsy or abnormal/unsatisfactory colposcopy during follow-up. Of the 108 patients who underwent a second excisional procedure or hysterectomy, 70 had negative findings, 10 had low-grade changes, 7 had CIN 2/3, 19 had AIS, and 2 had invasive adenocarcinoma (Table 2). Of the 19 cases with persistent AIS, 11 (57.9\%) had positive pathological margins in the original treatment specimen. There were 178 (59.7\%) patients that underwent cervical surveillance (cytology and/or cervical biopsy) which was negative in 151 patients, showed a low-grade abnormality in 24 and a highgrade abnormality in 3 patients for whom there were no data regarding a further 
excisional procedure. The remaining 12 patients did not have a cervical test result recorded in this period.

\section{Surveillance period ( $\geq 12$ months post treatment)}

After excluding the 28 patients that were treated with a second excisional procedure in the follow-up period-up and a further 39 patients who had a hysterectomy (hysterectomy specimen reported 35 cases as negative and 4 with low-grade changes), a total of 231 patients entered into the surveillance period. Of these 231 patients, $16(6.9 \%)$ patients underwent a second excisional procedure or hysterectomy ( $\geq 12$ months post treatment). Negative findings were recorded in 10 patients, 1 had low-grade changes, 2 had CIN 2/3 and 4 had recurrent AIS (Table 2). One patient that underwent a second excisional procedure for recurrent AIS was subsequently diagnosed with adenocarcinoma when a hysterectomy was performed. A further 18 patients had a cervical punch biopsy performed (12 cases were negative, 4 were low-grade changes and 2 with CIN2/3). A hysterectomy was performed for an additional 3 patients (all 3 cases were negative) in the surveillance period. A total of 162 patients underwent cervical surveillance (cervical screening) which was either

negative (147 patients) or showed only low-grade abnormalities (15 patients) and 32 patients did not have a cervical test result recorded in the surveillance period. Overall, $11(3.7 \%)$ patients had CIN 2/3, 23 (7.7\%) had AIS and $3(1.0 \%)$ had adenocarcinoma diagnosed during the follow-up and surveillance periods.

\section{Adenocarcinoma}

Overall there were 3 cases of invasive cervical adenocarcinoma detected in the followup and surveillance study periods. All 3 patients had a LEEP performed initially and 
their specimens were submitted in multiple fragments making pathological assessment difficult. In each case, extensive AIS was reported and AIS was reported to involve the biopsy margins. Pure AIS was present for 2 out of the 3 cancer cases. All patients subsequently underwent a second excisional procedure which reported the presence of adenocarcinoma.

Significant factors associated with disease persistence and recurrence A Kaplan-Meier graph shows that $10 \%$ of patients had disease persistence within 12 months of initial treatment (Figure 2). Kaplan-Meier graphs are also presented for margin status (Figure 3) and AIS lesion type (Figure 4).

Factors associated with disease persistence and recurrence were age $>30$ years at treatment (hazard rate ratio, 2.2; $95 \% \mathrm{CI}, 1.09-4.27$ ), a pure AIS lesion (hazard rate ratio, 3.2; $95 \% \mathrm{Cl}, 1.48-6.90$ ) and lesion size $>8 \mathrm{~mm}$ (hazard rate ratio, $4.8 ; 95 \% \mathrm{Cl}$, 1.21 - 18.92) (Table 3). Patient SEIFA index was not identified as significantly associated with disease persistence or recurrence and was excluded from the final statistical model.

\section{CONCLUSION}

To our knowledge, the present study is the largest investigation into outcomes for women with incidentally detected AIS following excisional treatment performed for high-grade CIN. Overall, persistent or recurrent high-grade in situ disease (AIS or CIN 2/3) was identified in $11.8 \%$ of the patient cohort [AIS, $(n=23)$ or CIN 2/3 $(n=$ 11)] and invasive adenocarcinomas in 3 cases (1.0\%) with almost all occurring within the first 12 months following the initial excisional procedure. 
Our findings are consistent with the results reported by Costa et al ${ }^{23}$, where older age was significantly associated with persistent/recurrent disease. A 2014 systematic review reported overall rates of AIS persistence/recurrence of $6-29 \%{ }^{24}$. In a Western Australian cohort of 338 patients treated for AIS between 2001 and 2012 with a median follow up interval of 3.6 years, AIS persistence/recurrence was reported in 27 women $(8 \%)^{5}$.

Up to $55 \%$ of cases AIS may co-exist with a high-grade squamous intraepithelial lesion$^{7}$. In the current study, pure AIS lesions (absence of co-existent CIN 2/3) detected incidentally on an excision specimen had an increased risk of disease persistence/recurrence when compared to mixed lesions (presence of co-existent CIN 2/3). This finding is notable and consistent with that of Song et al. who reported that patients diagnosed with a mixed cervical AIS lesion (CIN2/3 present) following cone biopsy had a more favorable prognosis than patients with pure $\mathrm{AIS}^{25}$.

An important finding in our study was that an AIS lesion $>8 \mathrm{~mm}$ in extent strongly correlated with persistence /recurrence. Consistent with the Canadian guidelines ${ }^{3}$, our findings suggest that smaller incidental AIS lesions $(<8 \mathrm{~mm})$ with clear margins do not require repeat excision, although this requires validation in larger prospective studies. Lesion size was recorded in only $51 \%$ of patients in our study because it is not mandatory for pathologists in Australia to report this. Prospective inclusion of AIS lesion size in excisional biopsy specimens would likely enhance our understanding of persistence/recurrence risk and inform future management guidelines. There were no 
significant differences in AIS persistence/recurrence by socioeconomic status, specimen depth and treatment modality (CKC versus LEEP).

Limitations of the current study include the selection bias inherent in its retrospective design, the relatively short median follow-up interval (2.4 years) and ascertainment bias. A further limitation is the absence of HPV data. However, legislative requirements for the reporting of cervical histopathology to the WA Registry would have resulted in the capture of almost all incident cases. Furthermore, the State-based Registry utilizes appropriate quality assurance processes to minimize error and ensure completeness of the data ${ }^{5,21}$. Valuable strengths of this study include the large size (298 cases) and population-based nature of the cohort.

In summary, following an incidental diagnosis of AIS, patient age $>30$ years, pure AIS lesions, and lesions $>8 \mathrm{~mm}$ in extent, were significantly associated with persistence/recurrence of AIS and/or CIN2/3. These findings require further validation, but future guidelines for the management of in situ cervical neoplasia might consider 'incidental AIS' as a separate category if it is demonstrated in prospective studies demonstrate this to have improved outcomes compared to cytologically detected AIS lesions.

In this study, incidental AIS was associated with a lower risk of persistence/recurrence. Hence younger women with incidental AIS lesions desiring conservative treatment, and especially those with co-existing with CIN 2/3 and AIS lesions $<8 \mathrm{~mm}$ in extent with clear margins, may not require immediate re-excision. Such patients should be counseled regarding the risk of AIS persistence/recurrence and the requirement to 
have colposcopy and a cervical screening test performed at 6 months after treatment (for 1 to 2 years) and then remain under surveillance with routine cervical screening and HPV testing $3,26,27$.

In older women, no longer desiring fertility preservation, hysterectomy may be considered as a definitive treatment option until prospective randomized data are available to better inform practice. 


\section{REFERENCES}

1. Andersen ES, Arffmann E. Adenocarcinoma in situ of the uterine cervix: a clinico-pathologic study of 36 cases. Gynecol Oncol. 1989; 35(1): 1-7.

2. Dunton CJ. Management of atypical glandular cells and adenocarcinoma in situ. Obstet Gyn Clin N Am. 2008; 35(4): 623-632.

3. Bentley J. Colposcopic management of abnormal cervical cytology and histology. J Obstet Gynaecol Can. 2012; 34(12): 1188-1206.

4. Kumar N, Bongiovanni M, Molliet MJ, Pelte MF, Egger JF, Pache JC. Diverse glandular pathologies coexist with high-grade squamous intraepithelial lesion in cyto-histological review of atypical glandular cells on ThinPrep specimens. Cytopathology. 2009; 20(6): 351-358.

5. Munro A, Leung Y, Spilsbury K, et al. Comparison of cold knife cone biopsy and loop electrosurgical excision procedure in the management of cervical adenocarcinoma in situ: What is the gold standard? Gynecol Oncol. 2015; 137(2): 258-263.

6. Kim J-H, Park J-Y, Kim D-Y, Kim Y-M, Kim Y-T, Nam J-H. The role of loop electrosurgical excisional procedure in the management of adenocarcinoma in situ of the uterine cervix. Eur J Obstet Gynecol Reprod Biol. 2009; 145(1): 100103.

7. Salani R, Puri I, Bristow RE. Adenocarcinoma in situ of the uterine cervix: a metaanalysis of 1278 patients evaluating the predictive value of conization margin status. Am J Obstet Gynecol. 2009; 200(2): 182 e1-5. 
8. Tierney KE, Lin PS, Amezcua C, et al. Cervical conization of adenocarcinoma in situ: a predicting model of residual disease. Am J Obstet Gynecol. 2014; 210(4): 366 e1-5.

9. National Medical Health and Research Council [NHMRC]. Screening to prevent cervical cancer: guidelines for the management of asymptomatic women with screen-detected abnormalities. Canberra; 2005.

10. Australian Institute of Health and Welfare [AlHW], Cancer Australia. Gynaecological cancers in Australia: an overview. Cancer series no. 70. Cat. no. CAN 66. Canberra: AlHW; 2012.

11. Australian Institute of Health and Welfare [AlHW]. Cervical screening in Australia 2013-2014. Cancer series no. 97. Cat. no. CAN 95. Canberra: AlHW; 2016.

12. van Hanegem N, Barroilhet LM, Nucci MR, Bernstein M, Feldman S. Fertilitysparing treatment in younger women with adenocarcinoma in situ of the cervix. Gynecol Oncol. 2012; 124(1): 72-77.

13. Latif NA, Neubauer NL, Helenowski IB, Lurain JR. Management of adenocarcinoma in situ of the uterine cervix: a comparison of loop electrosurgical excision procedure and cold knife conization. J Low Genit Tract Dis. 2015; 19(2): 97-102.

14. Bertrand M, Lickrish GM, Colgan TJ. The anatomic distribution of cervical adenocarcinoma in situ: implications for treatment. Am J Obstet Gynecol. 1987; 157(1): 21-25.

15. Jaworski RC. Endocervical glandular dysplasia, adenocarcinoma in situ, and early invasive (microinvasive) adenocarcinoma of the uterine cervix. Semin Diag Pathol. 1990; 7(3): 190-204. 
16. Loureiro J, Oliva E. The spectrum of cervical glandular neoplasia and issues in differential diagnosis. Arch Path Lab Med. 2014; 138(4): 453-483.

17. Östör AG, Duncan A, Quinn M, Rome R. Adenocarcinoma in situ of the uterine cervix: an experience with 100 Cases. Gynecol Oncol. 79(2): 207-210.

18. Bryson P, Stulberg R, Shepherd L, McLelland K, Jeffrey J. Is electrosurgical loop excision with negative margins sufficient treatment for cervical ACIS? Gynecol Oncol. 2004; 93(2): 465-468.

19. Holman CD, Bass AJ, Rosman DL, et al. A decade of data linkage in Western Australia: strategic design, applications and benefits of the WA data linkage system. Aust Health Rev. 2008; 32(4): 766-777.

20. Holman CD, Bass AJ, Rouse IL, Hobbs MS. Population-based linkage of health records in Western Australia: development of a health services research linked database. Aust N Z J Public Health. 1999; 23(5): 453-459.

21. Munro A, Williams V, Semmens J, et al. Risk of high-grade cervical dysplasia and gynaecological malignancies following the cytologic diagnosis of atypical endocervical cells of undetermined significance: a retrospective study of a state-wide screening population in Western Australia. Aust $N Z J$ Obstet Gynaecol. 2015; 55(3): 268-273.

22. Hirschowitz L, Ganesan R, Singh N, McCluggage WG. Standards and datasets for reporting cancers. Dataset for histological reporting of cervical neoplasia ( $3^{\text {rd }}$ edition). London: The Royal College of Pathologists; 2011.

23. Costa S, Venturoli S, Negri G, et al. Factors predicting the outcome of conservatively treated adenocarcinoma in situ of the uterine cervix: An analysis of 166 cases. Gynecol Oncol. 2012; 124(3): 490-495. 
24. Baalbergen A, Helmerhost TJ. Adenocarcinoma in situ of the uterine cervix-a systematic review. Int J Gynecol Cancer. 2014; 24(9): 1543-1548.

25. Song $\mathrm{T}$, Lee $\mathrm{YY}$, Choi $\mathrm{CH}$, et al. The effect of coexisting squamous cell lesions on prognosis in patients with cervical adenocarcinoma in situ. Eur J Obstet Gynecol Reprod Biol. 2015; 190(2015): 26-30.

26. Costa S, Venturoli S, Origoni M, et al. Performance of HPV DNA testing in the follow-up after treatment of high-grade cervical lesions, adenocarcinoma in situ (AIS) and microinvasive carcinoma. Ecancermedicalscience. 2015; 9:528.

27. Chan BK, Melnikow J, Slee CA, et al. Posttreatment human papillomavirus DNA testing for recurrent cervical intraepithelial neoplasia: a systematic review. Am J Obstet Gynecol Cancer. 2008; 200(4): 422.e1-422.e9. 